\title{
Accelerated hemithoracic radiation followed by extrapleural pneumonectomy for malignant pleural mesothelioma
}

\author{
Marc de Perrot, MD, MSc, ${ }^{a}$ Ronald Feld, MD, ${ }^{\mathrm{b}}$ Natasha B. Leighl, MD, ${ }^{\mathrm{b}}$ Andrew Hope, MD, \\ Thomas K. Waddell, MD, PhD, ${ }^{a}$ Shaf Keshavjee, $\mathrm{MD},{ }^{\mathrm{a}}$ and B. C. John Cho, MD, $\mathrm{PhD}^{\mathrm{c}}$
}

\begin{abstract}
Objective: To evaluate a new protocol of accelerated hemithoracic intensitymodulated radiation therapy (IMRT) followed by extrapleural pneumonectomy (EPP) for patients with resectable malignant pleural mesothelioma (MPM).

Methods: A total of 25 Gy of radiation was delivered in 5 daily fractions over 1 week to the entire ipsilateral hemithorax with concomitant boost of $5 \mathrm{~Gy}$ to volumes at high risk based on computed tomography and positron emission tomography scan findings. EPP was performed at $6 \pm 2$ days after the end of radiation therapy. Adjuvant chemotherapy was offered to patients with ypN2 disease.
\end{abstract}

Results: A total of 62 patients were included between November 2008 and October 2014. One patient died in the hospital 2 months after EPP, for an operative mortality of $1.6 \%$, and 2 died after discharged from the hospital for an overall treatment-related mortality (grade 5 toxicity) of $4.8 \%$. Twenty-four patients $(39 \%)$ developed grade 3 to 5 (grade $3+$ ) complications. On final pathology, $94 \%$ of the patients were stage III or IV, and $52 \%$ had ypN2 disease. The median survival for all patients as an intention-to-treat analysis was 36 months. The median overall survival and disease-free survival was 51 and 47 months, respectively, in epithelial subtypes, compared with 10 and 8 months in biphasic subtypes $(P=.001)$. Ipsilateral chest recurrence occurred in 8 patients.

Conclusions: Accelerated hemithoracic IMRT followed by EPP has become our preferred approach for resectable MPM. The results have been encouraging in patients with epithelial subtype. (J Thorac Cardiovasc Surg 2016;151:468-75)

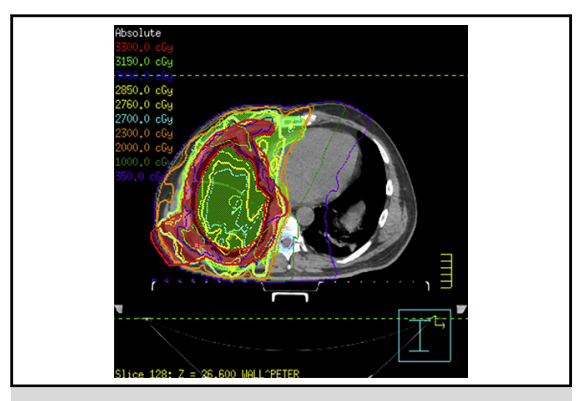

Surgery for mesothelioma after radiation therapy.

\section{Central Message}

Accelerated hemithoracic intensity-modulated radiation therapy followed by extrapleural pneumonectomy is feasible, and the results are encouraging for epithelial mesothelioma.

\section{Perspective \\ We present a new protocol of accelerated hemi- thoracic intensity-modulated radiation therapy followed by extrapleural pneumonectomy for patients with resectable malignant pleural mesothelioma.}

See Editorial Commentary page 476.

See Editorials page 307 and 310.
Although asbestos use has been regulated over the last 3 decades in industrialized countries, the burden of disease

From the Divisions of ${ }^{\mathrm{a}}$ Thoracic Surgery, ${ }^{\mathrm{b}}$ Medical Oncology, and ${ }^{\mathrm{c}}$ Department of Radiation Oncology, Princess Margaret Cancer Centre and Toronto General Hospital, University Health Network, University of Toronto, Toronto, Ontario, Canada.

This work was supported by the Princess Margaret Hospital Foundation Mesothelioma Research Fund.

ClinicalTrials.gov Identifier: NCT00797719.

Read at the 95th Annual Meeting of The American Association for Thoracic Surgery, Seattle, Washington, April 25-29, 2015.

Received for publication April 13, 2015; revisions received Aug 24, 2015; accepted for publication Sept 13, 2015; available ahead of print Nov 21, 2015.

Address for reprints: Marc de Perrot, MD, MSc, Toronto Mesothelioma Research Program, Toronto General Hospital, 9N-961, 200 Elizabeth St, Toronto, ON, Canada M5G 2C4 (E-mail: marc.deperrot@uhn.ca).

$0022-5223 / \$ 36.00$

Copyright $($ c 2016 by The American Association for Thoracic Surgery

http://dx.doi.org/10.1016/j.jtcvs.2015.09.129 related to its use continues to rise rapidly. ${ }^{1}$ In particular, malignant pleural mesothelioma (MPM) remains a major health problem. Despite aggressive therapy, including surgery, radiation, and chemotherapy, the prognosis of MPM remains extremely poor, with a median survival ranging between 17 and 20 months in 3 recent phase II prospective multi-institutional trials exploring the feasibility of a multimodality approach with induction chemotherapy followed by extrapleural pneumonectomy (EPP) and adjuvant hemithoracic radiation. ${ }^{2}$

Scanning this QR code will take you to the article title page. To view the AATS 2015 Webcast, see the URL at the end of the article. 


\section{Abbreviations and Acronyms \\ CT = Computed tomography \\ EBUS-TBNA $=$ Endobronchial ultrasound- transbronchial needle aspiration biopsy \\ EPD = Extended pleurectomy- decortication \\ $\mathrm{EPP}=$ Extrapleural pneumonectomy \\ FDG-PET = Fluorodeoxyglucose positron emission tomography \\ GTV = Gross tumor volume \\ IMRT $=$ Intensity modulated radiation therapy \\ MPM = Malignant pleural mesothelioma \\ SMART $=$ Surgery for mesothelioma after radiation therapy}

Encouraged by the improved rate of local control achieved with high-dose hemithoracic radiation in the adjuvant setting after EPP, we developed a new protocol of surgery for mesothelioma after radiation therapy (SMART). The rationale behind the development of this protocol was to optimize the delivery of radiation to the whole tumor bed, sterilize the edges of the tumor to limit the risk of spillage at the time of surgery, develop a shorter treatment plan, and potentiate activation of the immune system by using a hypofractionated regimen.

SMART entails a total of $25 \mathrm{~Gy}$ of radiation delivered in 5 daily fractions over 1 week to the entire ipsilateral hemithorax by intensity-modulated radiation therapy (IMRT), with a concomitant boost of $5 \mathrm{~Gy}$ to volumes at high risk based on computed tomography (CT) and positron emission tomography (PET) scan findings. EPP is performed within 2 weeks after the end of radiation therapy before the development of radiation pneumonitis. Adjuvant chemotherapy with cisplatin and an antifolate (pemetrexed or raltitrexed) doublet are administered selectively to patients with ypN2 disease on final pathology. The initial results of a seamless phase I/II trial demonstrated that this protocol is feasible and safe. ${ }^{3}$ The present analysis provides an update of our ongoing phase II expansion study with midterm outcome according to histological subtype and TNM status.

\section{MATERIALS AND METHODS}

Patients eligible for the SMART approach were at least 18 years of age and had an Eastern Cooperative Oncology Group performance status of 0 to 2, with good pulmonary function tests (defined as forced expiratory volume in 1 second $>40 \%$ predicted or diffusing capacity for carbon monoxide $>45 \%$ predicted), a new histological diagnosis of MPM previously untreated, clinical stage T1-3N0M0, suitable for combined modality therapy, and able to give informed consent. Clinical stage was determined by high-resolution CT scan of the chest and abdomen, fluorodeoxyglucose (FDG)-PET/CT scan, and brain magnetic resonance imaging or CT. Preoperative nodal sampling with endobronchial ultrasound-transbronchial needle aspiration biopsy (EBUS-TBNA) or mediastinoscopy was not performed routinely up to December 2013. Between November 2008 and December 2013, EBUS $(\mathrm{n}=6)$ or mediastinoscopy $(\mathrm{n}=2)$ was performed in 8 patients with enlarged paratracheal nodes to rule out $\mathrm{N} 2$ disease before entering the study. Starting in December 2013, EBUS became part of our routine staging to analyze the impact of this procedure on clinical staging, and thus the last 8 patients of the study had a negative EBUS result before entering the study. The clinical trial was approved by our hospital's Institutional Review Board.

Of note, patients were carefully evaluated before proceeding with induction radiation, to ensure that the tumor was resectable and to avoid the need for exploratory thoracotomy and the potential risk of fatal radiation pneumonitis. Patient selection was based on clinical symptoms, chest CT findings, and laboratory values. The imaging results were first reviewed to ensure that the patient had no evidence of chest wall, peritoneal, or mediastinal extension, particularly along the main bronchus. The clinical evaluation focused primarily on the degree of chest pain to ensure that it was limited and not requiring high doses of opioids. The laboratory values were also reviewed before starting radiation therapy, to ensure the absence of any major abnormality, particularly in the white blood cell, red blood cell, and platelet counts.

The clinical target volume was defined as the ipsilateral hemithorax, from the thoracic inlet down to the diaphragmatic insertion, including biopsy and drainage tract sites. The chest wall (ribs and intercostal muscles) was included in the radiation field. The gross tumor volume was defined as any tumor seen on CT and FDG-PET. The prescribed dosage to the clinical target volume was 25 Gy in 5 daily fractions over 1 week, with a concomitant boost of $5 \mathrm{~Gy}$ to the gross tumor volume and tract sites. A multibeam IMRT technique was used for all patients.

All patients underwent EPP within 2 weeks of completing neoadjuvant IMRT. Surgery was performed following a standard technique with resection and reconstruction of the diaphragm and pericardium. ${ }^{4}$ The bronchial stump was always covered either with the posterior pericardium, according to a technique that we recently described, or with a flap from the omentum or thymus.

In our initial cohort, partial wound dehiscence occurred in 4 of 25 patients $(16 \%)$ after surgery; a modification of our surgical technique has resolved this problem. ${ }^{3}$ In brief, the thoracotomy wound is closed with Ticron sutures (Covidien, Ontario, Canada) for the muscle and subcutaneous tissue layers. Each port site is radiated preoperatively to a diameter of $6 \mathrm{~cm}$ around the port site. Port sites are then resected only in the presence of gross disease, preserving the skin and subcutaneous tissue to ensure adequate closure without tension. The localized chest wall defect is closed with a small patch of 1-mm Gore-Tex mesh (W.L. Gore and Associates, Flagstaff, Ariz) to seal the pleural space and prevent the development of chest wall seroma.

Histological diagnosis and staging was based on the 2004 World Health Organization classification system and the seventh edition of the TNM staging system. ${ }^{6}$ Patients demonstrating mediastinal lymph node involvement on final pathology (ie, ypN2) were offered adjuvant chemotherapy with cisplatin combined with an antifolate agent, either raltitrexed or pemetrexed (at the discretion of the medical oncologist), for at least 3 cycles within 24 weeks after EPP.

After completing therapy, patients were followed at least every 3 months up to 2 years and then every 6 months up to 5 years. CT of the thorax and abdomen were performed at $3,6,12,18$, and 24 months and then yearly afterward. Additional tests were performed at the discretion of the investigators. Recurrences were diagnosed clinically, usually by serial imaging and proven pathologically when feasible. Recurrences were treated off protocol. Follow-up was completed up to October 2014.

The study was designed as an expansion study to assess efficacy following a seamless phase I/II study demonstrating the safety of this 


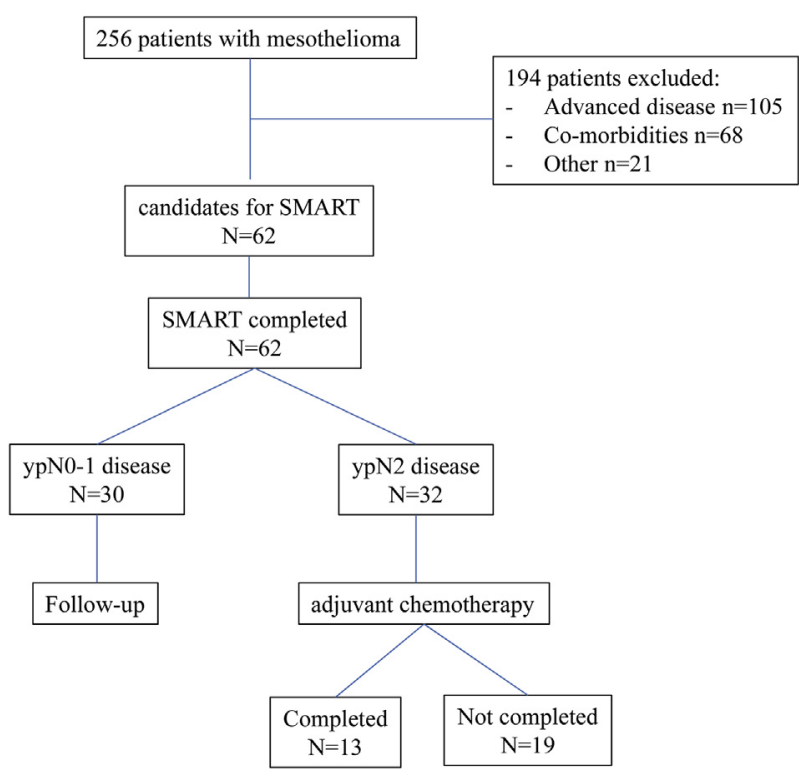

FIGURE 1. Consort diagram showing the flow of patients through the study. SMART, Surgery for mesothelioma after radiation therapy.

protocol. The seamless phase I/II study included 25 patients and was reported previously. ${ }^{3}$ The primary endpoint of the present expansion study was overall survival. Secondary endpoints included disease-free survival, treatment-related morbidity and mortality, and pattern of treatment failure. All patients who provided consent for the SMART protocol during the phase I/II study, the expansion study, and the transition period between both studies were recorded and followed prospectively in a similar fashion.

Demographic and treatment data, adverse event data, and survival data were reported as mean $\pm \mathrm{SD}$ or median and range. Categorical variables were compared using the $\chi^{2}$ test, and continuous variables were compared using the Student $t$ test. Treatment-related adverse events were reported

TABLE 1. Patient characteristics $(n=62)$

\begin{tabular}{lc}
\hline \multicolumn{1}{c}{ Characteristic } & Value \\
\hline Age, $\mathrm{y}$, median (range) & $64(41-75)$ \\
Sex, $\mathrm{n}(\%)$ & \\
$\quad$ Male & $52(84)$ \\
Female & $10(16)$ \\
Clinical stage, $\mathrm{n}(\%)$ & \\
T1N0 & $10(16)$ \\
T2N0 & $35(57)$ \\
T3N0 & $13(21)$ \\
T4N0 & $2(3)$ \\
T3N2 & $2(3)$ \\
Laterality, n (\%) & \\
Right & $45(73)$ \\
Left & $17(27)$ \\
Histology, n (\%) & \\
Epithelial & $44(71)$ \\
Biphasic & $18(29)$ \\
Chemotherapy, n (\%) & \\
None & $47(76)$ \\
Before SMART & $2(3)$ \\
Adjuvant (for ypN2) & $13(21)$ \\
\hline SMART Surgery for mesothelioma after radiation therapy
\end{tabular}

SMART, Surgery for mesothelioma after radiation therapy.
TABLE 2. Grade 3+ complications after SMART

\begin{tabular}{lcccc}
\hline & \multicolumn{4}{c}{ Complications } \\
\cline { 2 - 5 } \multicolumn{1}{c}{ Complication types } & Total & Grade 3 & Grade 4 & Grade 5 \\
\hline Patients with grade 3+ & 24 & & & \\
$\quad$ complications, $\mathrm{n}$ & & & & \\
Type of complication, $\mathrm{n} *$ & 12 & 12 & 0 & 0 \\
Atrial fibrillation & 4 & 2 & 1 & 1 \\
Empyema & 3 & 2 & 1 & 0 \\
Pulmonary emboli & 2 & 2 & 0 & 0 \\
Chylothorax & 2 & 0 & 2 & 0 \\
Hemothorax & 2 & 2 & 0 & 0 \\
Wound complications & 2 & 0 & 1 & 1 \\
Pneumonia & 1 & 1 & 0 & 0 \\
Renal dysfunction & 1 & 0 & 1 & 0 \\
Diaphragmatic patch dehiscence & 1 & 0 & 1 & 0 \\
Platypnea-orthodeoxia syndrome & 1 & 1 & 0 & 0 \\
Clostridium difficile colitis & 1 & 1 & \\
\hline
\end{tabular}

*Six patients had more than one grade $3+$ complication.

using the Common Terminology Criteria for Adverse Events, version 4.0. ${ }^{7}$ Overall survival was estimated using the Kaplan-Meier method. Survival was calculated from the starting day of radiation therapy for all patients, including 2 patients who underwent chemotherapy before radiation and surgery. Differences in survival were tested for significance using the log-rank test. Statview V (Abacus Concepts, Berkeley, Calif) was used for all analyses. A $P$ value $<.05$ was considered significant.

\section{RESULTS}

Of the 256 patients with MPM seen in our institution between November 2008 and October 2014, 62 (24\%) were deemed suitable candidates for the SMART approach (Figure 1). The vast majority of patients were males, with a median age of 64 years (Table 1). The clinical stage was T1NOM0 in 10 patients, T2NOM0 in 35 patients, and T3NOM0 in 13 patients. In 6 patients, the protocol was extended to include patients with tumor extending to the chest wall (cT4NOM0; $\mathrm{n}=2$ ), evidence of mediastinal lymph nodes involvement on PET scan (cT3N2M0; $\mathrm{n}=2$ ), or after completing 1 or 2 lines of chemotherapy $(n=2)$. One patient completed 6 cycles of cisplatinpemetrexed, and the second completed 6 cycles of cisplatin-pemetrexed, followed by 4 cycles of carboplatinpemetrexed, before starting the SMART protocol.

All 62 patients completed their intended IMRT and EPP. No patient dropped out between radiation and surgery. EPP was performed a mean of $6 \pm 2$ days after completion of IMRT. All but 1 patient underwent resection and reconstruction of the diaphragm, and all but 4 patients underwent resection and reconstruction of the pericardium. In 2 patients, a chest wall resection of 3 ribs was required to achieve complete macroscopic resection. Incomplete macroscopic resection (R2) was observed in 4 patients. The bronchial stump was covered by the posterior pericardium in 57 patients, by an omentum flap in 3 patients, and by a thymic flap in 2 patients. The median length of stay after surgery was 11 days (range, 5-102 days). 


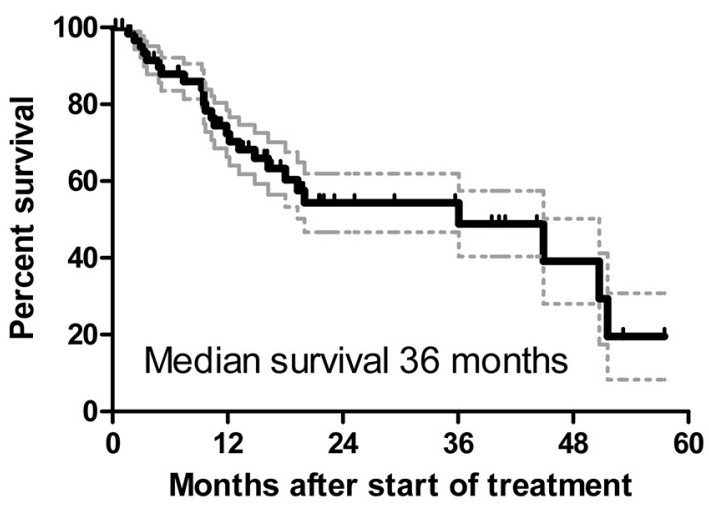

$\begin{array}{llllll}\text { Patients at risk } & 62 & 35 & 13 & 10 & 4\end{array}$

FIGURE 2. Overall survival as an intention-to-treat analysis for all 62 patients who started accelerated hemithoracic radiation therapy as part of the SMART protocol between November 2008 and October 2014.

A total of 24 patients (39\%) developed grade $3+$ complications (Table 2). The main complication was atrial fibrillation, occurring in 12 patients. Four patients developed an empyema, but none demonstrated evidence of bronchopleural fistula on further investigation. No patients died within 30 days of surgery.

Treatment-related death (grade 5 toxicity) occurred in 3 patients $(4.8 \%)$. One patient who underwent 10 cycles of chemotherapy before SMART developed an empyema and died in the hospital from pneumonia at 2 months after surgery, for a postoperative hospital mortality of $1.6 \%$. One patient who required chest wall resection and reconstruction for a biphasic mesothelioma was readmitted after discharge from the hospital with an empyema and died. A third patient died from an unwitnessed cardiac arrest at home.

On final pathology, 57 patients $(92 \%)$ presented with stage III $(\mathrm{n}=25)$ or IV $(\mathrm{n}=32)$, and 5 patients presented with stage I $(n=3)$ or II $(n=2)$. A total of 32 patients $(52 \%)$ had evidence of ypN2 disease on final pathology, and $3(5 \%)$ had ypN1 disease. Adjuvant chemotherapy was completed in $41 \%$ of the patients with ypN2 disease $(\mathrm{n}=13)$. The remaining patients with ypN2 disease did not undergo adjuvant chemotherapy owing to ongoing fatigue $(n=11)$, postoperative complications $(n=3)$, or early recurrence $(n=3)$. In addition, 1 patient started chemotherapy but stopped after a single cycle, and 1 patient with ypN2 disease completed chemotherapy before proceeding to SMART.

The current estimated median survival is 36 months, with 36 patients alive at last follow-up (Figure 2). No patient was lost to follow-up. The overall survival and disease-free survival were significantly better in patients with the epithelial subtype compared with those with the biphasic subtype (Figure 3). Among patients with cT1-3N0M0 previously untreated MPM $(\mathrm{n}=56)$, the median disease-
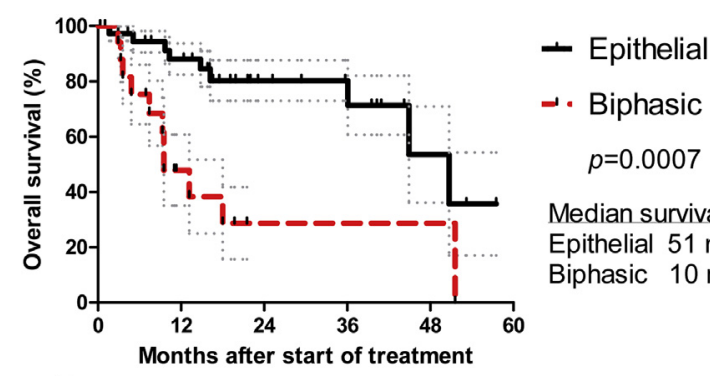

Median survival: Epithelial $51 \mathrm{mo}$ Biphasic $10 \mathrm{mo}$

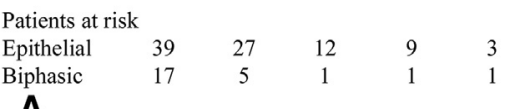

A

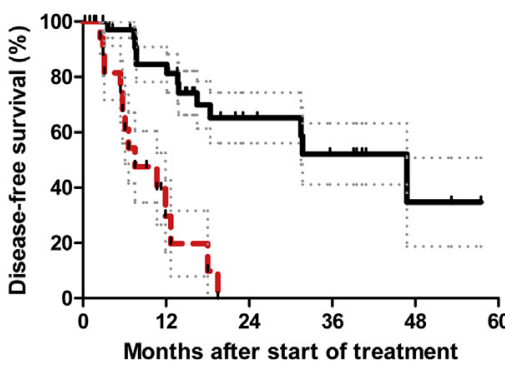

- Epithelial

- Biphasic

$p<0.0001$

Median DFS: Epithelial $47 \mathrm{mo}$ Biphasic $8 \mathrm{mo}$

Patients at risk

$\begin{array}{llllll}\text { Epithelial } & 39 & 26 & 11 & 7 & 2\end{array}$ Biphasic

B

FIGURE 3. Overall survival (A) and disease-free survival (B) according to histological subtype in 56 treatment-naive patients with cT1-3N0M0. Six patients with tumor extending to the chest wall on preoperative CT (cT4N0M0; $\mathrm{n}=2$ ), evidence of mediastinal lymph node involvement on PET scan (cT3N2M0; $\mathrm{n}=2$ ), or undergoing SMART after completing chemotherapy $(\mathrm{n}=2)$ were excluded from this analysis. $D F S$, disease free survival.

free survival was 47 months and overall survival was 51 months in patients with the epithelial subtype, compared to only 8 and 10 months, respectively, in patients with the biphasic subtype. Among patients with the epithelial subtype, the disease-free survival reached $66 \%$ at 3 years in patients with ypN0 disease, compared with $48 \%$ in patients with ypN + disease (Figure 4).

A total of 30 patients developed recurrence. The primary sites of recurrence were the contralateral chest $(n=9)$, the abdomen $(\mathrm{n}=8)$, and both the contralateral chest and abdomen $(\mathrm{n}=7)$. Abdominal recurrence was characterized by the presence of ascites $(n=7)$, retroperitoneal nodes $(n=5)$, peritoneal mass $(n=3)$, or liver mass $(n=2)$. Contralateral chest recurrence was characterized by parenchymal lung nodules $(\mathrm{n}=13)$ or pleural-based disease $(\mathrm{n}=3)$. Recurrence was seen within the ipsilateral chest in 8 patients, either alone $(n=5)$ or in combination with an abdominal recurrence $(n=3)$. Local recurrence in the ipsilateral chest was seen predominantly in patients with biphasic subtypes $(\mathrm{n}=5)$ and/or ypT4N2 disease $(\mathrm{n}=3)$. Other sites of recurrence included the pericardium $(n=2)$ and mediastinal lymph nodes $(\mathrm{n}=2)$ in combination with the contralateral chest recurrence. 


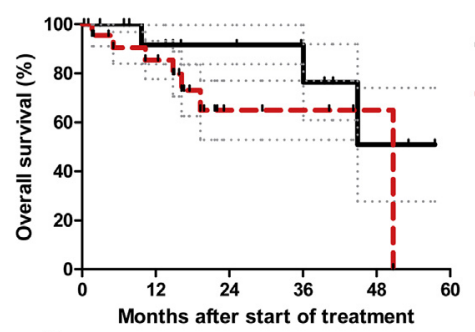

- Epithelial ypNO

$\leadsto$ Epithelial ypN+

$p=0.2$

Median survival: Epith ypNO not reached Epith ypN+ $51 \mathrm{mo}$

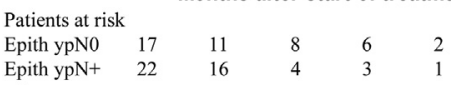
Epith ypN0
Epith ypN+

A

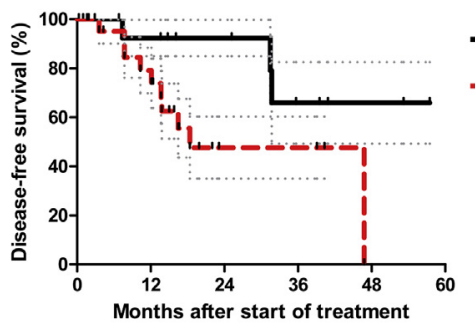

- Epithelial ypNO

$\sim$. Epithelial ypN+ $p=0.05$

Median DFS Epith ypNO not reached Epith ypN+ $18 \mathrm{mo}$

Patients at risk

$\begin{array}{llllll}\text { Epith ypN0 } & 17 & 11 & 8 & 4 & 2\end{array}$ Epith ypN+

B

FIGURE 4. Overall survival (A) and disease-free survival (B) in previously untreated cT1-3N0M0 patients with epithelial disease according to nodal status on final pathology. DFS, Disease free survival.

\section{DISCUSSION}

This study demonstrates that the SMART approach in patients with MPM is safe and compares favorably with other multimodality approach. In our experience, the overall survival as an intention-to-treat analysis was improved compared with our previous results with the trimodality approach using induction chemotherapy and adjuvant hemithoracic radiation after EPP. ${ }^{8}$ This analysis demonstrates that the SMART approach is particularly encouraging for patients with an epithelial subtype. In contrast, patients with a biphasic subtype had a dismal prognosis, with a median disease-free survival of only 8 months. Therefore, we currently exclude patients with a biphasic subtype from the SMART approach.

In our initial experience with the trimodality approach, we observed that adjuvant hemithoracic radiation achieved excellent local control and was potentially associated with improved survival in patients completing hemithoracic radiation in the absence of mediastinal nodal involvement. ${ }^{4,8}$ However, adjuvant hemithoracic radiation after 2 therapies (preoperative chemotherapy and surgery) was difficult to administer, and only one-half of the patients who started with induction chemotherapy completed the adjuvant radiation therapy. ${ }^{8}$ In addition, approximately $25 \%$ of the patients experienced disease progression during induction chemotherapy, thereby precluding them from surgery.

Encouraged by the results of adjuvant hemithoracic radiation on local control, ${ }^{9-11}$ as well as other evidence in the literature suggesting that MPM are radiosensitive

tumors, ${ }^{12}$ we designed this new trial to deliver radiation preoperatively to patients with surgically resectable tumors. Considering the risk of disease progression on induction chemotherapy, we felt that switching the order of therapy was potentially a better option for patients with surgically resectable disease, and thus started with preoperative radiation and reserving chemotherapy for the adjuvant setting. Given that the entire hemithorax including the lung had to be radiated, posing a risk of potentially fatal radiation pneumonitis, the radiation course was accelerated and EPP performed shortly thereafter. Adjuvant chemotherapy was proposed in the adjuvant setting for patients with ypN2 disease owing to the potentially poorer prognosis in these patients. However, this trial demonstrated once again the difficulty of administering a third therapy in this patient population, with only $41 \%$ of the patients with ypN2 disease completing the adjuvant chemotherapy.

Several institutions have used a similar protocol of accelerated radiotherapy for rectal cancer with excellent results. ${ }^{13,14} \mathrm{~A}$ recent randomized control trial in patients with rectal carcinoma found that a short course of induction radiation of $25 \mathrm{~Gy}$ in 5 daily fractions provided similar survival as a standard course of concurrent chemoradiation therapy followed by surgery. ${ }^{15}$ Considering the short interval between the end of radiation therapy and surgery, the goal of preoperative radiation is not to downstage the tumor, but rather theoretically to induce a tumorostatic and tumoricidal effect on the tumor to prevent or delay the successful implantation of metastasis to distant sites at the time of surgery and thereafter.

Over the past few years, the role of EPP in MPM has been increasingly called into question, ${ }^{16,17}$ and lung-sparing radical resection with extended pleurectomy-decortication (EPD) has become more popular. Several groups have developed intraoperative therapies in combination with radical surgery. Friedberg et $\mathrm{al}^{18}$ have reported good results by combining EPD with intraoperative photodynamic therapy, Sugarbaker et al ${ }^{19}$ have perfected the use of intraoperative hyperthermic chemotherapy, and Lang-Lazdunski et $\mathrm{al}^{20}$ have used hyperthermic povidone-iodine in combination with radical surgery. Despite the use of intraoperative therapy, EPD remains associated with a high rate of local recurrence compared with EPP, and some centers have begun to explore the possibility of adjuvant hemithoracic radiation after EPD to improve local control. ${ }^{21-23}$ This strategy has been associated with a risk of severe pneumonitis, however, and contributes to a decline in function of the preserved lung. ${ }^{22,24}$

The present study has some inherent limitations related to its design as a single-center trial with a single treatment arm. ${ }^{25}$ A longer follow-up is needed before definitive conclusions can be drawn about the success of this approach. Nonetheless, in our experience, this approach has been very encouraging and has become our primary option for patients with surgically resectable MPM. Owing to the lack of a clear 
benefit, patients with evidence of biphasic disease on the initial pleural biopsy or with clinical N2 disease are currently excluded from the SMART approach. This approach also carries the potential risk of severe toxicities, and thus we recommend that it remain confined to centers with extensive experience in hemithoracic radiation and surgery for MPM.

In conclusion, EPP can be performed after induction hemithoracic radiation therapy, and this approach is associated with encouraging overall survival and diseasefree survival in patients with epithelial cT1-3N0M0 mesothelioma. These encouraging results should support further studies to determine the role of hypofractionated radiation and surgery in the treatment of MPM.

\section{Conflict of Interest Statement}

Ronald Feld reports consulting fees from AstraZeneca Canada. Thomas K. Waddell reports consulting fees from United Therapeutics. Andrew Hope reports consulting and lecture fees from Elekta Inc. All other authors have nothing to disclose with regard to commercial support.

You can watch a Webcast of this AATS meeting presentation by going to: http://webcast.aats.org/2015/Video/ Monday/04-27-15_6C_1615_De_Perrot.mp4.

We thank Lea Dungao and Pat Merante for their assistance.

\section{References}

1. Stayner L, Welch LS, Lemen R. The worldwide pandemic of asbestos-related diseases. Annu Rev Public Health. 2013;34:205-16.

2. van Meerbeeck JP, Scherpereel A, Surmont VF, Baas P. Malignant pleural mesothelioma: the standard of care and challenges for future management. Crit Rev Oncol Hematol. 2011;78:92-111.

3. Cho BCJ, Feld R, Leighl N, Opitz I, Anraku M, Tsao MS, et al. A feasibility study evaluating surgery for mesothelioma after radiation therapy: the "SMART" approach for resectable malignant pleural mesothelioma. J Thorac Oncol. 2014;9:397-402.

4. de Perrot M, Uy K, Anraku M, Tsao MS, Darling G, Waddell TK, et al. Impact of lymph node metastasis on outcome after extrapleural pneumonectomy for malignant pleural mesothelioma. J Thorac Cardiovasc Surg. 2007;133:111-6.

5. de Perrot M. Use of the posterior pericardium to cover the bronchial stump after right extrapleural pneumonectomy. Ann Thorac Surg. 2013;96:706-8.

6. Travis WD, Brambilla E, Müller-Hermelink HK, Harris CC, eds. Pathology and Genetics: Tumours of the Lung, Pleura, Thymus and Heart. Lyon, France: IARC Press; 2004.

7. National Cancer Institute. National Cancer Institute Common Terminology Criteria for Adverse Events (CTCAE) v.4.0.2010. Available at: http://evs.nci. nih.gov/ftp1/CTCAE/About.html. Accessed November 3, 2015.

8. de Perrot M, Feld R, Cho BC, Bezjak A, Anraku M, Burkes R, et al. Trimodality therapy with induction chemotherapy followed by extrapleural pneumonectomy and adjuvant high-dose hemithoracic radiation for malignant pleural mesothelioma. J Clin Oncol. 2009;27:1413-8.

9. Rusch VW, Rosenzweig K, Venkatraman E, Leon L, Raben A, Harrison L, et al. A phase II trial of surgical resection and adjuvant high-dose hemithoracic radiation for malignant pleural mesothelioma. J Thorac Cardiovasc Surg. 2001;122:788-95.

10. Rice DC, Stevens CW, Correa AM, Vaporciyan AA, Tsao A, Forster KM, et al. Outcomes after extrapleural pneumonectomy and intensity-modulated radiation therapy for malignant pleural mesothelioma. Ann Thorac Surg. 2007;84:1685-92.

11. Buduhan G, Menon S, Aye R, Louie B, Mehta V, Vallières E. Trimodality therapy for malignant pleural mesothelioma. Ann Thorac Surg. 2009;88:870-5.

12. Jenkins $P$, Milliner R, Salmon C. Re-evaluating the role of palliative radiotherapy in malignant pleural mesothelioma. Eur J Cancer. 2011;47:2143-9.

13. Swedish Rectal Cancer Trial. Improved survival with preoperative radiotherapy in resectable rectal cancer. $N$ Engl J Med. 1997;336:980-7.
14. Minsky BD, Rödel C, Valentini V. Short-course radiation versus long-course chemoradiation for rectal cancer. J Natl Compr Canc Netw. 2012;10:1223-31.

15. Ngan SY, Burmeister B, Fisher RJ, Solomon M, Goldstein D, Joseph D, et al. Randomized trial of short-course radiotherapy versus long-course chemoradiation comparing rates of local recurrence in patients with T3 rectal cancer: TransTasman Radiation Oncology Group Trial 01.04. J Clin Oncol. 2012;30:3827-33.

16. Treasure T, Lang-Lazdunski L, Waller D, Bliss JM, Tan C, Entwisle J, et al. Extrapleural pneumonectomy versus no extra-pleural pneumonectomy for patients with malignant pleural mesothelioma: clinical outcomes of the Mesothelioma and Radical Surgery (MARS) randomised feasibility study. Lancet Oncol. 2011;12:763-72.

17. Weder W, Stahel RA, Baas P, Dafni U, de Perrot M, McCaughan BC, et al. The MARS feasibility trial: conclusions not supported by data. Lancet Oncol. 2011; 12:1093-4.

18. Friedberg JS, Culligan MJ, Mick R, Stevenson J, Hahn SM, Sterman D, et al. Radical pleurectomy and intraoperative photodynamic therapy for malignant pleural mesothelioma. Ann Thorac Surg. 2012;93:1658-65.

19. Sugarbaker DJ, Gill RR, Yeap BY, Wolf AS, DaSilva MC, Baldini EH, et al Hyperthermic intraoperative pleural cisplatin chemotherapy extends interval to recurrence and survival among low-risk patients with malignant pleural mesothelioma undergoing surgical macroscopic complete resection. J Thorac Cardiovasc Surg. 2013;145:955-63.

20. Lang-Lazdunski L, Bille A, Papa S, Marshall S, Lal R, Galeone C, et al. Pleurectomy/decortication, hyperthermic pleural lavage with povidone-iodine, prophylactic radiotherapy, and systemic chemotherapy in patients with malignant pleural mesothelioma: a 10-year experience. J Thorac Cardiovasc Surg. 2015;149:558-66.

21. Rimner A, Spratt DE, Zauderer MG, Rosenzweig KE, Wu AJ, Foster A, et al. Failure patterns after hemithoracic pleural intensity-modulated radiation therapy for malignant pleural mesothelioma. Int J Radiat Oncol Biol Phys. 2014;90:394-401.

22. Chance WW, Rice DC, Allen PK, Tsao AS, Fontanilla HP, Liao Z, et al. Hemithoracic intensity-modulated radiation therapy after pleurectomy/ decortication for malignant pleural mesothelioma: toxicity, patterns of failure, and a matched survival analysis. Int J Radiat Oncol Biol Phys. 2015;91:149-56.

23. Minatel E, Trovo M, Polesel J, Baresic T, Bearz A, Franchin G, et al. Radical pleurectomy/decortication followed by high dose of radiation therapy for malignant pleural mesothelioma: final results with long-term follow-up. Lung Cancer. 2014;83:78-82.

24. Rosenzweig KE, Zauderer MG, Laser B, Krug LM, Yorke E, Sima CS, et al Pleural intensity-modulated radiotherapy for malignant pleural mesothelioma. Int J Radiat Oncol Biol Phys. 2012;83:1278-83.

25. Treasure T, Utley M. Ten traps for the unwary in surgical series: a case study in mesothelioma reports. J Thorac Cardiovasc Surg. 2007;133:1414-8.

Key Words: mesothelioma, MPM, short course radiation, surgery

\section{Discussion}

Dr D. Rice (Houston, Tex). Marc, I want to congratulate you on an excellent presentation and thanks for getting me the manuscript well in advance of this meeting.

As you know, the surgical management of this disease is very controversial, and generally poor outcomes with surgery alone have prompted people to use bimodality and trimodality regimens, which is why we have used postoperative hemithoracic radiation, and this has translated into good local control but hasn't resulted really in better long-term survival. Importantly, it is feasible in only approximately two-thirds of the patients who we want to administer it in. It can cause significant morbidity, especially IMRT, especially with respect to late pulmonary events.

You present a new paradigm for delivery of radiation in this disease, and, importantly, the short-course technique that you describe is able to be successfully administered to all patients without any significant toxicity, and 
subsequently extrapleural pneumonectomy, not a trivial operation, was able to be performed safely, with extremely low perioperative mortality. Your local recurrence rate is comparable to what we see with adjuvant radiation techniques, but the median survival is excellent, 36 months. I think this represents probably the best long-term results of any cytoreductive series that I am aware of. I am particularly impressed, since over $92 \%$ of your patients had stage III or IV disease.

I have 3 questions. First of all, could you elaborate on the radiation technique and the dosage? Particularly, what is the biological effective dose, and what was the contralateral lung dose, V20, and were there any late radiation-related pulmonary events in the contralateral lung?

Dr de Perrot. Thank you very much, David, for your comments.

The biological effective dose of 25 to $30 \mathrm{~Gy}$ in 5 fractions correlates to about 45 to $48 \mathrm{~Gy}$ in a more standard way to deliver the radiation. It is not a radical dose, but it is a dose that is acceptable in combination with surgery. That specific type of radiation has been used in rectal carcinoma in the past. That is why we developed our own protocol. In rectal carcinoma, the accelerated induction radiation to 25 Gy was similar to chemoradiation therapy before surgery with similar outcomes, again, in rectal carcinoma. Obviously, we are still closely monitoring the long-term effect of the radiation. So far, we haven't seen long term side effects from the radiation, but it is a high dose and clearly it has to be monitored closely.

Dr Rice. My second question pertains to the fact that, like others, you have shown an extremely high rate of N2 disease. Given the fact that the minority of patients underwent any kind of preoperative histological assessment, have you changed your practice in preoperative staging of these patients?

Dr de Perrot. Yes. Initially these patients were based on CT scan, on PET CT scan, CT scan of the chest and abdomen. The nodal status clearly has an impact, mostly in T4N2 disease, but we are currently staging our patients with EBUS. All of our patients have EBUS in addition to $\mathrm{CT}$ and PET scan currently.

Dr Rice. My last question is, and I know you probably don't have data to support this, but what do you think is going on? Your median survival here is twice what you reported with the previous induction regimen with chemotherapy, surgery, and radiation. Is this an effect of better local control? Is it an immunotherapeutic effect? I would like your opinion.

I really want to congratulate you for advancing the treatment of this disease. We really need a little bit of good news on mesothelioma, so thank you.

Dr de Perrot. Thank you.

The effect is still something that we are trying to understand better. Certainly, one potential impact of this type of radiation is on the immune system. We have had animal models of mesothelioma in our lab, and over the past few years, based on the findings in our clinical trial, we have developed an animal model looking at hypofractionated radiation in mice, and there is clearly an immunogenic effect of the high-dose short-course radiation on the immune system, which potentially opens the door to using that type of radiation in combination with immunomodulatory agents. That is something that may develop in the future, I believe.

Dr D. Sugarbaker (Houston, Tex). I have a couple of questions. First, it was a very nice paper, very well presented, and kind of exciting data.

What was your resectability rate? How many patients did you open and you could not resect?

Dr de Perrot. There were no patients that we opened and did not resect. These are patients who were selected preoperatively to make sure that they could undergo the extrapleural pneumonectomy based on the fact that if they had radiation and no surgery, they would have severe pneumonitis. The operability rate of patients that we saw in our clinic was about $24 \%$. So $24 \%$ of the patients we have seen in the clinic were included in that trial.

Dr Sugarbaker. So you are selecting patients who you obviously can assess are $100 \%$ resectable? Most studies, including ours, would suggest that with good radiographic evidence, you can get about an $80 \%$ to $85 \%$ resectability rate. That is the first question.

The second question is, when you say that $94 \%$ of these patients were stage III, are you going by the TNM staging system, the one currently published? I take it that is your staging system. Is that right?

Dr de Perrot. Yes.

Dr Sugarbaker. The third question I have is, do you have any idea what the tumor volume was in these patients? From what you have told me, it sounds like you are operating on relatively low-volume disease to ensure a $100 \%$ resectability rate. Is that fair?

Dr de Perrot. It is certainly a selected group of patients, there is no question about it, and because we had more experience with the protocol, we extended the indications. We certainly have been careful, particularly at the beginning. But I will say that over the 7-year period, initially we selected patients who had either trimodality therapy with induction chemo followed by EPP and radiation, and progressively switched to include all surgical patients

Dr Sugarbaker. I understand, but basically what you are saying is that you put patients in this trial who you could virtually guarantee were resectable?

Dr de Perrot. Yes.

Dr Sugarbaker. And in mesothelioma, that means that you are really being extremely conservative as to who you operate on, because there are patients who you think are clearly resectable, who biologically, when you get in there, are not. As I say, I think the best you can get is about an $80 \%$ resectability rate. I 
mean it's fine, but it sounds like it's really early-stage disease or, put it this way, low tumor volume.

Dr de Perrot. Yes, the tumor volume is certainly something that we are looking at to get a better idea of the selection of the patients. Certainly this is a selected group of patients and it requires experience and this is an evolving process, again, as we have shown over the past 7 years in our institution, but once you select the patients, they need to have the surgery, and, again, I think it was about $24 \%$ of the patients that we saw over the course of the 7 years who underwent that protocol. That is relatively similar to our prior experience with the trimodality approach, where it was about $25 \%$ of the patients who were treated with EPP after the chemotherapy with adjuvant radiation. So I think with increasing experience, we have selected more or less the same patients, with the exclusion of N2 disease on the PET scan mainly. Again, I would certainly agree with you that this is a selected group of patients, and you have to be very careful to select the right patients to make sure they can have the surgery, and with increasing experience, you can make sure progressively that you can resect these patients in epithelial disease. In biphasic disease, occasionally you find much more locally advanced disease than you would expect on the CT scan, and currently we do not include biphasic disease based on the outcome that I showed you.

Dr R. Flores (New York, NY). I have a word of caution and a question. First is a word of caution when you have a small number of patients, 62 patients, for mesothelioma. When you have longer follow-up and a greater number of patients, you are probably going to see a difference in that median survival. As shown by your Kaplan-Meier curve, the majority of patients are grouped together in the earlier parts of the graph. I also echo what Dr Sugarbaker says. Whenever you have a group of mesothelioma patients who have a zero unresectability rate, that raises a red flag. My question is, did you look at the pathological specimens of the epithelioid patients and see if there was a response in those specimens?

Dr de Perrot. The time frame between the end of the radiation and the surgery is about 6 days. This is too short to really see an effect histologically. There was no downstaging on the pathological evaluation. That doesn't mean that there was no effect on the tumor. It's just that the tumor is affected in the sense that the cells are not viable cells, and in the xenograft model that we have in the lab, if you implant these tumors after radiation, the vast majority of them do not grow. So I do believe there is an effect of the radiation, and I think our data to some extent supports that. But I certainly agree with you that this is a selected group of patients. This is too early to make a final commitment as to what the long-term effect of that trial is. Also, this is a type of treatment that carries potential significant risk, and I certainly would think that it should be limited to institutions that have a large experience in hemithoracic radiation, at least in the adjuvant setting, and also a large experience in treating mesothelioma surgically to make sure that these are patients who can have surgery, who can have a resection, an EPP, once you stop the preoperative radiation. I think it is too early to make a final conclusion, and I certainly will.

Dr Sugarbaker. Marc, I have to cut you off. We need to get to these other folks.

Dr D. Harpole (Durham, NC). Marc, very nice use of hypofractionated radiation as an induction regimen.

My only question is sort of a follow-up on what Dr Sugarbaker was asking. In our hands, we certainly have on the order of about 1 in 4, 1 in 5 patients who get in there and they either have tumor involving the esophagus or some of the structures, that we have not at that time performed an extrapleural and we have converted to a pleurectomy, and they were patients who we thought had limited disease and that we could do this. So if I were to do that now, I've got a lung that has now had hypofractionated radiation to it. I'm asking you, what should I do? I'm very concerned now that I have got a lung that is going to have severe radiation injury. What would be your suggestion for me?

Dr de Perrot. First of all, if you start that protocol, you have to resect the lung, even if it looks difficult. Sometimes when you start the surgery very early in the morning, you wonder how you are going to finish the day, but at the end of the day, you have to resect the lung. Two patients had a chest wall resection, and that is part of what you are facing, is to be able to resect the lung. But, again, this is experience that you build up over time. When we started, we certainly selected patients who had earlier stage. I think for people who will look at using that protocol, clearly you have to be very careful that you can resect the lungs initially, and as you build up experience, I think you can become more aggressive. Also, that protocol is for early stages. Obviously you cannot choose that protocol for more advanced disease. So it's a balance. It is a selected group, and all our patients were resectable based on the initial assessment, but I think once you don't have a choice, you can finish the surgery with an EPP.

Dr V. Rusch (New York, NY). Congratulations on very novel work. As you have pointed out, this treatment approach is not yet exportable beyond highly experienced centers, and must still be considered investigational. You have raised an interesting issue of whether there is still a role for EPP in highly selected patients. The patterns of disease progression are similar to what has been reported in other series of EPP plus adjuvant hemithoracic radiation, with patients primarily experiencing disease progression in distant sites. It will be interesting to see the results of your work on the immunogenicity of this approach, because it may prove to be responsible for better survival in this group of patients.

Dr de Perrot. Thank you. I certainly agree with all of your comments. 